elderly suicide rates and life expectancy and markers of socio-economic status and healthcare status. International Psychogeriatrics, 20, 347-360.

Stack, S. (1993). The effect of modernization on suicide in Finland, 1800-1985. Sociological Perspective, 36, 137-148.

doi:10.1017/\$1041610208007539

\section{The relationship between elderly suicide rates and the affordability of psychotropic drugs}

A recent cross-national study of 62 developing and developed countries reported an increase in suicide rates with aging in males and females in 25 and 27 countries respectively (Shah, 2007). Longitudinal studies over time have reported a decline in elderly suicide rates in England and Wales (Gunnell et al., 2003; Lodhi and Shah, 2004), Sweden (Carlsten et al., 1999) and Australia (Hall et al., 2003) with an increase in prescription rates of antidepressants, particularly selective serotonin reuptake inhibitors. The vast majority of elderly suicide victims have depressive illness (Shah and De, 1998). The pathway to elderly depressed individuals acquiring a prescription of antidepressants includes the following sequential steps: recognition by individuals or their carers that there is problem and consulting a healthcare professional; recognition of depression by the healthcare professional; prescription of antidepressants by the healthcare professional; and, purchasing of the antidepressants by the patient. Different methods of acquiring the prescribed antidepressants exist in different countries including: healthcare professionals dispensing the antidepressant free or at a cost; and obtaining the prescribed antidepressant from a pharmacist free (either through state welfare system or insurance schemes) or at a cost. We have examined the relationship between elderly suicide rates and the affordability of psychotropic medication in a cross-national study.

Two unidirectional hypotheses were tested: (1) there would be a negative correlation between elderly suicide rates and affordability of antidepressants given a strong relationship between depression and elderly suicide; and (2) there would be no association between elderly suicide rates and affordability of anti-dementia drugs given the unequivocal relationship between dementia and elderly suicides. One null hypothesis was tested: there would be no relationship between elderly suicide rates and affordability of antipsychotic drugs given a weak association between schizophrenia and elderly suicides.

\author{
AJIT SHAH ${ }^{1}$ AND RITESH BHANDARKAR ${ }^{2}$ \\ ${ }^{1}$ Centre for Ethnicity and Health, University of Central \\ Lancashire, Preston, U.K. \\ ${ }^{2}$ West London Mental Health NHS Trust, London, U.K. \\ Email: ajit.shah@wlmht.nhs.uk
}

The International Psychogeriatric Association's Mental Health Economic Task Force, led by Professor G. K. Suh, has recently produced data on the affordability of psychotropic drugs in 20 countries as measured by the affordability index (AI). The AI is a measure of the number of tablets of a particular psychotropic medication at a particular dose that can be purchased with one day's income. Data on the AI were available for the following drugs for different preparations and doses: anti-dementia drugs (donepezil, galanthamine, rivastigmin and memantine); antidepressants (moclobemide, citalopam, sertraline, paroxetine, mirtazepine, venlofaxine and bupropion); and antipsychotics (amisulpiride, aripiprazole, clozapine, olanzapine, risperidone, quetiapine and zis).

Data on suicide rates for males and females for the general population and specifically in the age-bands 65-74 years and 75+ years were ascertained from the WHO website (www.who.int/ whosis/database/mort/table1.cfm) for the latest available year. Spearman's rank correlation test $(\rho)$ was used to examine the relationship between suicide rates for males and females in the general population and specifically in the age-bands $65-74$ years and $75+$ years and the AI index for all the different doses and preparations of all the psychotropic drugs listed above.

Data on both the AI and suicide rates were available for a median (range) of 9 (2-15) countries depending on the precise psychotropic drug, dose and preparation (not all doses and all drugs were available in all the countries). There was no significant correlation between $\mathrm{AI}$ and suicide rates in any group with three exceptions. There was a positive correlation between the male general population suicide rate and the AI of clozapine $25 \mathrm{mg}(\rho=0.76, \mathrm{p}=0.006)$ and clozapine $100 \mathrm{mg}(\rho=0.71, \mathrm{p}=0.022)$; in females this relationship was only seen with clozapine $100 \mathrm{mgs}(\rho=0.63, \mathrm{p}=0.039)$. The significance of these correlations disappeared when corrections for multiple comparisons were applied.

The absence of correlation between AI and elderly suicide rates is not consistent with earlier reports of a decline in elderly suicide rates with an increase in prescription rates of antidepressants 
in England and Wales (Gunnell et al., 2003; Lodhi and Shah, 2004), Sweden (Carlsten et al., 1999) and Australia (Hall et al., 2003). There may be several reasons for this. First, the sample size was small. Second, some countries may use older antidepressants for which data on the AI were absent. Third, the AI does not take into account awareness of depression among individuals and their carers, identification of depression by healthcare professionals, appropriateness of treatment for depression offered by healthcare professionals, and compliance for taking antidepressants by patients. Fourth, the AI does not take account of different payment systems for prescriptions: in some countries healthcare professionals may dispense medication directly without charging the patient; in countries with welfare systems elderly patients may be exempt from paying directly for their prescription; and, in other countries, prescription costs may be covered by insurance. Fifth, treatments such as cognitive behavior therapy may be offered in some countries instead of antidepressants.

If the AI for all antidepressants can be calculated for all the countries where the patient has to pay for the antidepressant prescription at the time of presenting the prescription then the true relationship between elderly suicide rates and the AI of antidepressants can be examined.

doi:10.1017/S1041610208007722

\section{Apathy is a syndrome of executive dysfunction that exists in patients with late-life depression}

Marin et al. (2003) have suggested that apathy may be independent of executive functions in olderaged patients with major depression, although the correlation between apathy and the "controlled oral word association test," a test of executive measures, approached significance. However, Feil et al. (2003), using the same definition and measure of apathy, observed a strong correlation between apathy and both verbal and non-verbal executive cognitive measures. The question thus arises as to whether apathy contributes to executive cognitive dysfunction in non-demented patients with late-life depression. We therefore re-examined this issue, looking particularly at whether apathy distinctly affects executive control functions. Our hypothesis was that apathy would contribute to cognitive impairment in depressed elderly patients independently of the severity of depression.

In our prospective cohort study, we enrolled 84 patients from the geriatric psychiatric clinic of the Chang Gung Memorial Hospital with major

\section{References}

Carlsten, A., Waern, M. and Allebeck, P. (1999). Suicides by drug poisoning among the elderly in Sweden 1969-1996. Social Psychiatry and Psychiatric Epidemiology, 34, 609-614.

Gunnell, D., Middleston, N., Whitley, E., Doring, D. and Frankel, S. (2003). Why are suicide rates rising in young men but falling in the elderly? A time-series analysis of trends in England and Wales, 1950-1998. Social Science and Medicine, 57, 595-611.

Hall, W., Mant, A., Mitchell, P., Rendle, V. A., Hickie, I. B. and McManus, P. (2003). Association between antidepressant prescribing and suicide in Australia, 1991-2000: trend analysis. BMF, 326, 1008-1011.

Lodhi, L. and Shah, A. K. (2004). Psychotropic prescriptions and elderly suicide rates. Medicine, Science and the Law, 44, 236-244.

Shah, A. K. (2007). The relationship between suicide rates and age: an analysis of multinational data from the World Health Organization. International Psychogeriatrics, 19, 1141-1152.

Shah, A. K. and De, T. (1998). Suicide in the elderly. International fournal of Psychiatry in Clinical Practice, 2, 3-17.
AJit SHAH ${ }^{1}$ AND GURLEEN Bhatia ${ }^{2}$
${ }^{1}$ Professor of Ageing, Ethnicity and Mental Health, University of Central Lancashire,
Preston and Consultant Psychiatrist, West London
Mental Health NHS Trust, London, U.K.
Email: ajit.shah@wlmht.nhs.uk
${ }^{2}$ Trainee Psychiatrist, West London Mental Health NHS Trust, London, UK.

depressive disorder. The inclusion criteria included a diagnosis of unipolar major depression based on DSM-IV. The exclusion criteria included a diagnosis of dementia of the Alzheimer's type or vascular dementia based on the DSM-IV criteria. Patients with a history of any psychiatric disorders other than major depression, or neurological disorders that may influence cognition, were also excluded. All participants were administered the 17-item Hamilton rating scale for depression (HamD) to evaluate their depression status. Among these Ham-D items, the apathy-related items included diminished work/interest, anergy, lack of insight, and psychomotor retardation. These items showed a robust correlation with the Apathy Evaluation Scale (Marin et al., 1991) and ranged in score from 0 to 12 . We therefore used the apathy-related items of the Ham-D as a proxy measure of apathy.

We employed pairs of behavioral tasks to assess whether apathy has a distinct effect on executive control functions. Each pair of tests consisted of an executive and a non-executive task. First, we assessed verbal fluency as an executive cognitive measure with object naming as a non-executive control function. Second, we examined backward digit span as an executive cognitive measure with 